\title{
New inequalities for $F$-convex functions pertaining generalized fractional integrals
}

\author{
Hüseyin Budak, Pinar Kösem, Artion Kashuri
}

\begin{abstract}
In this paper, the authors, utilizing $F$-convex functions which are defined by B. Samet, establish some new Hermite-Hadamard type inequalities via generalized fractional integrals. Some special cases of our main results recaptured the well-known earlier works.
\end{abstract}

\section{INTRODUCTION}

Let $f: I \subseteq R \rightarrow R$ be a convex function on the interval $I$ of real numbers and $a, b \in I$ with $a<b$. If $f$ is a convex function then the following double inequality, which is well known in the literature as the Hermite-Hadamard inequality, holds [17]:

$$
f\left(\frac{a+b}{2}\right) \leq \frac{1}{b-a} \int_{a}^{b} f(x) \mathrm{d} x \leq \frac{f(a)+f(b)}{2} .
$$

Both inequalities in (1) hold in the reversed direction if $f$ is concave.

Over the last decade, this classical double inequality has been improved and generalized in a number of ways, see [5, 7, 8, 13, 18], [23]-[25] and the references therein. Also, many types of convexities have been defined, such as quasi-convex in [6], pseudo-convex in [14], strongly convex in [20], $\varepsilon$-convex in [11], $s$-convex in [10], $h$-convex in [28], etc. Recently, Samet in [21], has defined a new concept of convexity that depends on a certain function satisfying some axioms, that generalizes different types of convexity.

Recall the family $\mathcal{F}$ of mappings $F: \mathbb{R} \times \mathbb{R} \times \mathbb{R} \times[0,1] \rightarrow \mathbb{R}$ satisfying the following axioms:

2010 Mathematics Subject Classification. Primary: 26D07; Secondary: 26D10, 26D15, $26 \mathrm{~A} 33$.

Key words and phrases. Convex function, trigonometrically $\rho$-convex functions.

Full paper. Received 9 December 2019, revised 21 July 2020, accepted 28 July 2020, available online 16 September 2020. 
(A1) If $e_{i} \in L^{1}(0,1), i=1,2,3$, then for every $\lambda \in[0,1]$, we have

$$
\int_{0}^{1} F\left(e_{1}(t), e_{2}(t), e_{3}(t), \lambda\right) \mathrm{d} t=F\left(\int_{0}^{1} e_{1}(t) \mathrm{d} t, \int_{0}^{1} e_{2}(t) \mathrm{d} t, \int_{0}^{1} e_{3}(t) \mathrm{d} t, \lambda\right) ;
$$

(A2) For every $u \in L^{1}(0,1), w \in L^{\infty}(0,1)$ and $\left(z_{1}, z_{2}\right) \in \mathbb{R}^{2}$, we have

$$
\int_{0}^{1} F\left(w(t) u(t), w(t) z_{1}, w(t) z_{2}, t\right) \mathrm{d} t=T_{F, w}\left(\int_{0}^{1} w(t) u(t) \mathrm{d} t, z_{1}, z_{2}\right),
$$

where $T_{F, w}: \mathbb{R} \times \mathbb{R} \times \mathbb{R} \rightarrow \mathbb{R}$ is a function that depends on $(F, w)$, and it is nondecreasing with respect to the first variable;

(A3) For any $\left(w, e_{1}, e_{2}, e_{3}\right) \in \mathbb{R}^{4}, e_{4} \in[0,1]$, we have

$$
w F\left(e_{1}, e_{2}, e_{3}, e_{4}\right)=F\left(w e_{1}, w e_{2}, w e_{3}, e_{4}\right)+L_{w},
$$

where $L_{w} \in \mathbb{R}$ is a constant that depends only on $w$.

Definition 1. Let $f:[a, b] \rightarrow \mathbb{R},(a, b) \in \mathbb{R}^{2}, a<b$, be a given function. We say that $f$ is a convex function with respect to some $F \in \mathcal{F}$ (or $F$-convex function), if and only if:

$$
F(f(t x+(1-t) y), f(x), f(y), t) \leq 0, \quad(x, y, t) \in[a, b] \times[a, b] \times[0,1] .
$$

Remark 1. 1) Let $\varepsilon \geq 0$, and let $f:[a, b] \rightarrow \mathbb{R},(a, b) \in \mathbb{R}^{2}, a<b$, be an $\varepsilon$-convex function, see [11], that is

$$
f(t x+(1-t) y) \leq t f(x)+(1-t) f(y)+\varepsilon, \quad(x, y, t) \in[a, b] \times[a, b] \times[0,1] .
$$

Define the functions $F: \mathbb{R} \times \mathbb{R} \times \mathbb{R} \times[0,1] \rightarrow \mathbb{R}$ by

$$
F\left(e_{1}, e_{2}, e_{3}, e_{4}\right)=e_{1}-e_{4} e_{2}-\left(1-e_{4}\right) e_{3}-\varepsilon
$$

and $T_{F, w}: \mathbb{R} \times \mathbb{R} \times \mathbb{R} \rightarrow \mathbb{R}$ by

$$
T_{F, w}\left(e_{1}, e_{2}, e_{3}\right)=e_{1}-\left(\int_{0}^{1} t w(t) \mathrm{d} t\right) e_{2}-\left(\int_{0}^{1}(1-t) w(t) \mathrm{d} t\right) e_{3}-\varepsilon
$$

For

$$
L_{w}=(1-w) \varepsilon
$$

it is clear that $F \in \mathcal{F}$ and $F(f(t x+(1-t) y), f(x), f(y), t)=f(t x+(1-t) y)-t f(x)-(1-t) f(y)-\varepsilon \leq 0$, that is $f$ is an $F$-convex function. Particularly, taking $\varepsilon=0$, we show that if $f$ is a convex function then $f$ is an $F$-convex function with respect to $F$ defined above. 
2) Let $h: J \rightarrow[0,+\infty)$ be a given function which is not identical to 0 , where $J$ is an interval in $\mathbb{R}$ such that $(0,1) \subseteq J$. Let $f:[a, b] \rightarrow[0,+\infty)$, $(a, b) \in \mathbb{R}^{2}, a<b$, be an $h$-convex function, see [28], that is

$f(t x+(1-t) y) \leq h(t) f(x)+h(1-t) f(y), \quad(x, y, t) \in[a, b] \times[a, b] \times[0,1]$.

Define the functions $F: \mathbb{R} \times \mathbb{R} \times \mathbb{R} \times[0,1] \rightarrow \mathbb{R}$ by

$$
F\left(e_{1}, e_{2}, e_{3}, e_{4}\right)=e_{1}-h\left(e_{4}\right) e_{2}-h\left(1-e_{4}\right) e_{3}
$$

and $T_{F, w}: \mathbb{R} \times \mathbb{R} \times \mathbb{R} \rightarrow \mathbb{R}$ by

(6) $T_{F, w}\left(e_{1}, e_{2}, e_{3}\right)=e_{1}-\left(\int_{0}^{1} h(t) w(t) \mathrm{d} t\right) e_{2}-\left(\int_{0}^{1} h(1-t) w(t) \mathrm{d} t\right) e_{3}$.

For $L_{w}=0$, it is clear that $F \in \mathcal{F}$ and

$F(f(t x+(1-t) y), f(x), f(y), t)=f(t x+(1-t) y)-h(t) f(x)-h(1-t) f(y) \leq 0$, that is, $f$ is an $F$-convex function.

Samet in [21], established the following Hermite-Hadamard type inequalities using the new convexity concept:

Theorem 1. Let $f:[a, b] \rightarrow \mathbb{R},(a, b) \in \mathbb{R}^{2}, a<b$, be an $F$-convex function, for some $F \in \mathcal{F}$. Suppose that $f \in L^{1}[a, b]$. Then

$$
\begin{gathered}
F\left(f\left(\frac{a+b}{2}\right), \frac{1}{b-a} \int_{a}^{b} f(x) \mathrm{d} x, \frac{1}{b-a} \int_{a}^{b} f(x) \mathrm{d} x, \frac{1}{2}\right) \leq 0, \\
T_{F, 1}\left(\frac{1}{b-a} \int_{a}^{b} f(x) \mathrm{d} x, f(a), f(b)\right) \leq 0 .
\end{gathered}
$$

Definition 2. Let $f \in L^{1}[a, b]$. The Riemann-Liouville integrals $J_{a+}^{\alpha} f$ and $J_{b-}^{\alpha} f$ of order $\alpha>0$ are defined by

$$
J_{a+}^{\alpha} f(x)=\frac{1}{\Gamma(\alpha)} \int_{a}^{x}(x-t)^{\alpha-1} f(t) \mathrm{d} t, \quad x>a
$$

and

$$
J_{b-}^{\alpha} f(x)=\frac{1}{\Gamma(\alpha)} \int_{x}^{b}(t-x)^{\alpha-1} f(t) \mathrm{d} t, \quad x<b,
$$

respectively. Here, $\Gamma(\alpha)$ is the Gamma function and

$$
J_{a+}^{0} f(x)=J_{b-}^{0} f(x)=f(x) .
$$

Definition 3. Let $f \in L^{1}[a, b]$. Then $k$-fractional integrals of order $\alpha, k>0$ are defined by

$$
I_{a^{+}, k}^{\alpha} f(x)=\frac{1}{k \Gamma_{k}(\alpha)} \int_{a}^{x}(x-t)^{\frac{\alpha}{k}-1} f(t) \mathrm{d} t, \quad x>a,
$$


and

$$
I_{b^{-}, k}^{\alpha} f(x)=\frac{1}{k \Gamma_{k}(\alpha)} \int_{x}^{b}(t-x)^{\frac{\alpha}{k}-1} f(t) \mathrm{d} t, \quad b>x,
$$

where $\Gamma_{k}(\cdot)$ stands for the $k$-gamma function. For $k=1$, the $k$-fractional integrals yield Riemann-Liouville integrals. For $\alpha=k=1$, the $k$-fractional integrals yield classical integrals. For more details, see [9, 12, 15, 19].

It is remarkable that Sarikaya et al. in [26], first give the following interesting integral inequalities of Hermite-Hadamard type involving RiemannLiouville fractional integrals.

Theorem 2. Let $f:[a, b] \rightarrow \mathbb{R}$ be a positive function with $0 \leq a<b$ and $f \in L^{1}[a, b]$. If $f$ is a convex function on $[a, b]$, then the following inequalities for fractional integrals hold:

$$
f\left(\frac{a+b}{2}\right) \leq \frac{\Gamma(\alpha+1)}{2(b-a)^{\alpha}}\left[J_{a+}^{\alpha} f(b)+J_{b-}^{\alpha} f(a)\right] \leq \frac{f(a)+f(b)}{2},
$$

with $\alpha>0$.

Budak et al. in [1], prove the following Hermite-Hadaamrd type inequalities for $F$-convex functions via fractional integrals:

Theorem 3. Let $I \subseteq R$ be an interval, $f: I^{\circ} \subseteq \mathbb{R} \rightarrow \mathbb{R}$ be a mapping on $I^{\circ}, a, b \in I^{\circ}, a<b$. If $f$ is $F$-convex on $[a, b]$ for some $F \in \mathcal{F}$, then we have

$$
\begin{aligned}
F & \left(f\left(\frac{a+b}{2}\right), \frac{\Gamma(\alpha+1)}{(b-a)^{\alpha}} J_{a^{+}}^{\alpha} f(b), \frac{\Gamma(\alpha+1)}{(b-a)^{\alpha}} J_{b^{-}}^{\alpha} f(a), \frac{1}{2}\right) \\
& +\int_{0}^{1} L_{w(t)} \mathrm{d} t \leq 0,
\end{aligned}
$$

and

$$
\begin{aligned}
T_{F, w} & \left(\frac{\Gamma(\alpha+1)}{(b-a)^{\alpha}}\left[J_{a^{+}}^{\alpha} f(b)+J_{b^{-}}^{\alpha} f(a)\right], f(a)+f(b), f(a)+f(b)\right) \\
+ & \int_{0}^{1} L_{w(t)} \mathrm{d} t \leq 0,
\end{aligned}
$$

where $w(t)=\alpha t^{\alpha-1}$.

For other papers involving $F$-convex functions, see [1]-[4], [16, 27].

Now we summarize the generalized fractional integrals defined by Sarikaya and Ertuğral in [22].

Let's define a function $\varphi:[0,+\infty) \rightarrow[0,+\infty)$ satisfying the following conditions:

$$
\int_{0}^{1} \frac{\varphi(t)}{t} \mathrm{~d} t<+\infty
$$




$$
\begin{gathered}
\frac{1}{A_{1}} \leq \frac{\varphi(v)}{\phi(u)} \leq A_{1} \text { for } \frac{1}{2} \leq \frac{v}{u} \leq 2, \\
\frac{\varphi(u)}{u^{2}} \leq A_{2} \frac{\varphi(v)}{v^{2}} \text { for } v \leq u, \\
\left|\frac{\varphi(u)}{u^{2}}-\frac{\varphi(v)}{v^{2}}\right| \leq A_{3}|u-v| \frac{\varphi(u)}{u^{2}} \text { for } \frac{1}{2} \leq \frac{v}{u} \leq 2,
\end{gathered}
$$

where $A_{1}, A_{2}, A_{3}>0$ are independent of $u, v>0$. If $\varphi(u) u^{\alpha}$ is increasing for some $\alpha \geq 0$ and $\frac{\varphi(u)}{u^{\beta}}$ is decreasing for some $\beta \geq 0$, then $\varphi$ satisfies the above conditions.

The following left-sided and right-sided generalized fractional integral operators are defined respectively, as follows:

$$
\begin{aligned}
& a^{+} I_{\varphi} f(x)=\int_{a}^{x} \frac{\varphi(x-t)}{x-t} f(t) \mathrm{d} t, \quad x>a, \\
& { }^{-} I_{\varphi} f(x)=\int_{x}^{b} \frac{\varphi(t-x)}{t-x} f(t) \mathrm{d} t, \quad x<b .
\end{aligned}
$$

The most important feature of generalized fractional integrals is that they generalize some types of fractional integrals such as Riemann-Liouville fractional integral, $k$-Riemann-Liouville fractional integral, Katugampola fractional integrals, conformable fractional integral, Hadamard fractional integrals, etc.

Sarikaya and Ertuğral in [22], establish the following Hermite-Hadamard inequality and lemmas for the generalized fractional integral operators:

Theorem 4. Let $f:[a, b] \rightarrow \mathbb{R}$ be a convex function on $[a, b]$ with $a<b$, then the following inequalities for fractional integral operators hold:

$$
f\left(\frac{a+b}{2}\right) \leq \frac{1}{2 \Psi(1)}\left[a+I_{\varphi} f(b)+{ }_{b-} I_{\varphi} f(a)\right] \leq \frac{f(a)+f(b)}{2},
$$

where the mapping $\Lambda:[0,1] \rightarrow \mathbb{R}$ is defined by

$$
\Psi(x)=\int_{0}^{x} \frac{\varphi((b-a) t)}{t} \mathrm{~d} t .
$$

Budak et al. prove the following Hermite Hadamard type inequalities for $F$-convex functions.

Theorem 5 ([4]). Let $I \subseteq \mathbb{R}$ be an interval, $f: I^{\circ} \subseteq \mathbb{R} \rightarrow \mathbb{R}$ be a mapping on $I^{\circ}, a, b \in I^{\circ}, a<b$. If $f$ is $F$-convex on $[a, b]$ for some $F \in \mathcal{F}$, then we have

$$
F\left(f\left(\frac{a+b}{2}\right), \frac{1}{\Psi(1)}{ }_{a+} I_{\varphi} f(b), \frac{1}{\Psi(1)}{ }_{b-} I_{\varphi} f(a), \frac{1}{2}\right)+\int_{0}^{1} L_{w(t)} \mathrm{d} t \leq 0,
$$


and

$$
\begin{aligned}
& T_{F, w}\left(\frac{1}{\Psi(1)}\left[{ }_{a+} I_{\varphi} f(b)+{ }_{b-} I_{\varphi} f(a)\right], f(a)+f(b), f(a)+f(b)\right) \\
& \quad+\int_{0}^{1} L_{w(t)} \mathrm{d} t \leq 0,
\end{aligned}
$$

where $w(t)=\frac{\varphi((b-a) t)}{t \Psi(1)}$.

Motivated by the above literatures, the main objective of this article is to establish some new Hermite-Hadamard type inequalities via generalized fractional integrals utilizing $F$-convex functions. Some special cases of our main results recaptured the well-known earlier works. At the end, a briefly conclusion will be given as well.

\section{MAIN RESUlts}

In this section, we establish some inequalities of Hermite-Hadamard type including generalized fractional integrals via $F$-convex functions.

Theorem 6. Let $I \subseteq \mathbb{R}$ be an interval, $f: I^{\circ} \subseteq \mathbb{R} \rightarrow \mathbb{R}$ be a mapping on $I^{\circ}$, $a, b \in I^{\circ}, a<b$ and let $F$ be linear with respect to the first three variables. If $f$ is $F$-convex on $[a, b]$ for some $F \in \mathcal{F}$, then we have

$$
\begin{aligned}
F & \left(f\left(\frac{a+b}{2}\right), \frac{1}{\Lambda(1)}\left(\frac{a+b}{2}\right)^{+} I_{\varphi} f(b), \frac{1}{\Lambda(1)}\left(\frac{a+b}{2}\right)^{-} I_{\varphi} f(a), \frac{1}{2}\right) \\
& +\int_{0}^{1} L_{w(t)} \mathrm{d} t \leq 0,
\end{aligned}
$$

and

$$
\begin{gathered}
T_{F, w}\left(\frac { 1 } { \Lambda ( 1 ) } \left[\left(\frac{a+b}{2}\right)^{+} I_{\varphi} f(b)+\left(\frac{a+b}{2}\right)^{\left.-I_{\varphi} f(a)\right]},\right.\right. \\
f(a)+f(b), f(a)+f(b))+\int_{0}^{1} L_{w(t)} \mathrm{d} t \leq 0,
\end{gathered}
$$

where $w(t)=\frac{\varphi\left(\left(\frac{b-a}{2}\right) t\right)}{t \Lambda(1)}$ and the function $\Lambda:[0,1] \rightarrow \mathbb{R}$ is defined by

$$
\Lambda(x)=\int_{0}^{x} \frac{\varphi\left(\frac{b-a}{2} t\right)}{t} \mathrm{~d} t .
$$

Kanıt. Since $f$ is $F$-convex, we have

$$
F\left(f\left(\frac{x+y}{2}\right), f(x), f(y), \frac{1}{2}\right) \leq 0, \quad \forall x, y \in[a, b] .
$$


For

we have

$$
x=\frac{t}{2} a+\left(\frac{2-t}{2}\right) b \text { and } y=\left(\frac{2-t}{2}\right) a+\frac{t}{2} b,
$$

$$
F\left(f\left(\frac{a+b}{2}\right), f\left(\frac{t}{2} a+\left(\frac{2-t}{2}\right) b\right), f\left(\left(\frac{2-t}{2}\right) a+\frac{t}{2} b\right), \frac{1}{2}\right) \leq 0 .
$$

for all $t \in[0,1]$. Multiplying this inequality by $w(t)=\frac{\varphi\left(\left(\frac{b-a}{2}\right) t\right)}{t \Lambda(1)}$ and using axiom (A3), we get

$$
\begin{gathered}
F\left(\frac{\varphi\left(\left(\frac{b-a}{2}\right) t\right)}{t \Lambda(1)} f\left(\frac{a+b}{2}\right), \frac{\varphi\left(\left(\frac{b-a}{2}\right) t\right)}{t \Lambda(1)} f\left(\frac{t}{2} a+\left(\frac{2-t}{2}\right) b\right),\right. \\
\left.\frac{\varphi\left(\left(\frac{b-a}{2}\right) t\right)}{t \Lambda(1)} f\left(\left(\frac{2-t}{2}\right) a+\frac{t}{2} b\right), \frac{1}{2}\right)+L_{w(t)} \leq 0
\end{gathered}
$$

for all $t \in(0,1)$. Integrating over $(0,1)$ with respect to the variable $t$ and using axiom (A1), we obtain

$$
\begin{gathered}
F\left(\frac{f\left(\frac{a+b}{2}\right)}{\Lambda(1)} \int_{0}^{1} \frac{\varphi\left(\frac{(b-a)}{2} t\right)}{t} \mathrm{~d} t, \frac{1}{\Lambda(1)} \int_{0}^{1} \frac{\varphi\left(\frac{(b-a)}{2} t\right)}{t} f\left(\frac{t}{2} a+\left(\frac{2-t}{2}\right) b\right) \mathrm{d} t,\right. \\
\left.\frac{1}{\Lambda(1)} \int_{0}^{1} \frac{\varphi\left(\frac{(b-a)}{2} t\right)}{t} f\left(\left(\frac{2-t}{2}\right) a+\frac{t}{2} b\right) \mathrm{d} t, \frac{1}{2}\right)+\int_{0}^{1} L_{w(t)} \mathrm{d} t \leq 0 .
\end{gathered}
$$

Using the facts that

$$
\begin{aligned}
& \int_{0}^{1} \frac{\varphi\left(\frac{(b-a)}{2} t\right)}{t} f\left(\frac{t}{2} a+\left(\frac{2-t}{2}\right) b\right) \mathrm{d} t \\
= & \int_{\frac{a+b}{2}}^{b} \frac{\varphi(b-x)}{b-x} f(x) \mathrm{d} x={ }_{\left(\frac{a+b}{2}\right)^{+}} I_{\varphi} f(b)
\end{aligned}
$$

and

$$
\begin{aligned}
& \int_{0}^{1} \frac{\varphi\left(\frac{(b-a)}{2} t\right)}{t} f\left(\left(\frac{2-t}{2}\right) a+\frac{t}{2} b\right) \mathrm{d} t \\
= & \int_{a}^{\frac{a+b}{2}} \frac{\varphi(x-a)}{x-a} f(x) \mathrm{d} x={ }_{\left(\frac{a+b}{2}\right)^{-}} I_{\varphi} f(a),
\end{aligned}
$$

we obtain

$$
\begin{aligned}
F & \left(f\left(\frac{a+b}{2}\right), \frac{1}{\Lambda(1)}\left(\frac{a+b}{2}\right)^{+} I_{\varphi} f(b), \frac{1}{\Lambda(1)}\left(\frac{a+b}{2}\right)^{-} I_{\varphi} f(a), \frac{1}{2}\right) \\
& +\int_{0}^{1} L_{w(t)} \mathrm{d} t \leq 0,
\end{aligned}
$$


which gives (18).

On the other hand, since $f$ is $F$-convex, we have

$$
F\left(f\left(\frac{t}{2} a+\left(\frac{2-t}{2}\right) b\right), f(a), f(b), t\right) \leq 0, \quad \forall t \in[0,1],
$$

and

$$
F\left(f\left(\left(\frac{2-t}{2}\right) a+\frac{t}{2} b\right), f(a), f(b), t\right) \leq 0, \quad \forall t \in[0,1]
$$

Using the linearity of $F$, we get

$$
\begin{gathered}
F\left(f\left(\frac{t}{2} a+\left(\frac{2-t}{2}\right) b\right)+f\left(\left(\frac{2-t}{2}\right) a+\frac{t}{2} b\right),\right. \\
f(a)+f(b), f(a)+f(b), t) \leq 0,
\end{gathered}
$$

for all $t \in[0,1]$. Applying the axiom (A3) for $w(t)=\frac{\varphi\left(\frac{(b-a)}{2} t\right)}{t \Lambda(1)}$, we obtain

$$
\begin{aligned}
F & \left(\frac{\varphi\left(\frac{(b-a)}{2} t\right)}{t \Lambda(1)}\left[f\left(\frac{t}{2} a+\left(\frac{2-t}{2}\right) b\right)+f\left(\left(\frac{2-t}{2}\right) a+\frac{t}{2} b\right)\right],\right. \\
& \left.\frac{\varphi\left(\frac{(b-a)}{2} t\right)}{t \Lambda(1)}[f(a)+f(b)], \frac{\varphi\left(\frac{(b-a)}{2} t\right)}{t \Lambda(1)}[f(a)+f(b)], t\right)+L_{w(t)} \leq 0,
\end{aligned}
$$

for all $t \in(0,1)$. Integrating over $(0,1)$ and using axiom (A2), we have

$$
\begin{gathered}
T_{F, w}\left(\int_{0}^{1} \frac{\varphi\left(\frac{(b-a)}{2} t\right)}{t \Lambda(1)}\left[f\left(\frac{t}{2} a+\left(\frac{2-t}{2}\right) b\right)+f\left(\left(\frac{2-t}{2}\right) a+\frac{t}{2} b\right)\right] \mathrm{d} t,\right. \\
f(a)+f(b), f(a)+f(b))+\int_{0}^{1} L_{w(t)} \mathrm{d} t \leq 0,
\end{gathered}
$$

that is

$$
\begin{aligned}
& T_{F, w}\left(\frac{1}{\Lambda(1)}\left[\left(\frac{a+b}{2}\right)^{+} I_{\varphi} f(b)+\left(\frac{a+b}{2}\right)^{-} I_{\varphi} f(a)\right], f(a)+f(b), f(a)+f(b)\right) \\
& \quad+\int_{0}^{1} L_{w(t)} \mathrm{d} t \leq 0 .
\end{aligned}
$$

The proof of Theorem 6 is completed. 
Remark 2. If we choose $\varphi(t)=t$ in Theorem 6 , then we have the following inequalities

$$
\begin{aligned}
& F\left(f\left(\frac{a+b}{2}\right), \frac{2}{b-a} \int_{\frac{a+b}{2}}^{b} f(t) \mathrm{d} t, \frac{2}{b-a} \int_{a}^{\frac{a+b}{2}} f(t) \mathrm{d} t, \frac{1}{2}\right) \\
& \quad+\int_{0}^{1} L_{w(t)} \mathrm{d} t \leq 0,
\end{aligned}
$$

and

$$
T_{F, w}\left(\frac{2}{b-a} \int_{a}^{b} f(t) \mathrm{d} t, f(a)+f(b), f(a)+f(b)\right)+\int_{0}^{1} L_{w(t)} \mathrm{d} t \leq 0,
$$

where $w(t)=1$.

Remark 3. If we choose $\varphi(t)=\frac{t^{\alpha}}{\Gamma(\alpha)}$ in Theorem 6, then we have the following inequalities for Riemann-Liouville fractional integrals

$$
\begin{aligned}
& F\left(f\left(\frac{a+b}{2}\right), \frac{2^{\alpha} \Gamma(\alpha+1)}{(b-a)^{\alpha}} J_{\left(\frac{a+b}{2}\right)^{+}}^{\alpha} f(b), \frac{2^{\alpha} \Gamma(\alpha+1)}{(b-a)^{\alpha}} J_{\left(\frac{a+b}{2}\right)^{-}}^{\alpha} f(a), \frac{1}{2}\right) \\
& +\int_{0}^{1} L_{w(t)} \mathrm{d} t \leq 0,
\end{aligned}
$$

and

$$
\begin{gathered}
T_{F, w}\left(\frac{2^{\alpha} \Gamma(\alpha+1)}{(b-a)^{\alpha}}\left[J_{\left(\frac{a+b}{2}\right)^{+}}^{\alpha} f(b)+J_{\left(\frac{a+b}{2}\right)^{-}}^{\alpha} f(a)\right],\right. \\
f(a)+f(b), f(a)+f(b))+\int_{0}^{1} L_{w(t)} \mathrm{d} t \leq 0,
\end{gathered}
$$

where $w(t)=\alpha t^{\alpha-1}$ which is given by Budak et al. in [5].

Corollary 1. If we take $\varphi(t)=\frac{t^{\frac{\alpha}{k}}}{k \Gamma_{k}(\alpha)}$ in Theorem 6, then we have the following inequalities for $k$-Riemann-Liouville fractional integrals

$$
\begin{aligned}
F\left(f\left(\frac{a+b}{2}\right), \frac{2^{\frac{\alpha}{k}} \Gamma_{k}(\alpha+k)}{(b-a)^{\frac{\alpha}{k}}} I_{\left(\frac{a+b}{2}\right)+, k}^{\alpha} f(b),\right. \\
\\
\left.\quad \frac{2^{\frac{\alpha}{k}} \Gamma_{k}(\alpha+k)}{(b-a)^{\frac{\alpha}{k}}} I_{\left(\frac{a+b}{2}\right)-, k}^{\alpha} f(a), \frac{1}{2}\right)+\int_{0}^{1} L_{w(t)} \mathrm{d} t \leq 0,
\end{aligned}
$$

and

$$
T_{F, w}\left(\frac{2^{\frac{\alpha}{k}} \Gamma_{k}(\alpha+k)}{(b-a)^{\frac{\alpha}{k}}}\left[I_{\left(\frac{a+b}{2}\right)+, k}^{\alpha} f(b)+I_{\left(\frac{a+b}{2}\right)-, k}^{\alpha} f(a)\right],\right.
$$




$$
f(a)+f(b), f(a)+f(b))+\int_{0}^{1} L_{w(t)} \mathrm{d} t \leq 0,
$$

where $w(t)=\frac{\alpha}{k} t^{\frac{\alpha}{k}-1}$.

Theorem 7. Let $I \subseteq \mathbb{R}$ be an interval, $f: I^{\circ} \subseteq \mathbb{R} \rightarrow \mathbb{R}$ be a mapping on $I^{\circ}$, $a, b \in I^{\circ}, a<b$ and let $F$ be linear with respect to the first three variables. If $f$ is $F$-convex on $[a, b]$ for some $F \in \mathcal{F}$, then we have

$$
\begin{aligned}
F & \left(f\left(\frac{a+b}{2}\right), \frac{1}{\Lambda(1)} b_{-} I_{\varphi} f\left(\frac{a+b}{2}\right),\right. \\
& \left.\frac{1}{\Lambda(1)}{ }_{a+} I_{\varphi} f\left(\frac{a+b}{2}\right), \frac{1}{2}\right)+\int_{0}^{1} L_{w(t)} \mathrm{d} t \leq 0,
\end{aligned}
$$

and

$$
\begin{gathered}
T_{F, w}\left(\frac{1}{\Lambda(1)}\left[{ }_{a+} I_{\varphi} f\left(\frac{a+b}{2}\right)+{ }_{b-} I_{\varphi} f\left(\frac{a+b}{2}\right)\right],\right. \\
f(a)+f(b), f(a)+f(b))+\int_{0}^{1} L_{w(t)} \mathrm{d} t \leq 0,
\end{gathered}
$$

where $w(t)=\frac{\varphi\left(\left(\frac{b-a}{2}\right) t\right)}{t \Lambda(1)}$.

Kanıt. Since $f$ is $F$-convex, we have

$$
F\left(f\left(\frac{x+y}{2}\right), f(x), f(y), \frac{1}{2}\right) \leq 0, \quad \forall x, y \in[a, b] .
$$

For

$$
x=\left(\frac{1-t}{2}\right) a+\left(\frac{1+t}{2}\right) b \text { and } y=\left(\frac{1+t}{2}\right) a+\left(\frac{1-t}{2}\right) b,
$$

we have

$$
\begin{gathered}
F\left(f\left(\frac{a+b}{2}\right), f\left(\left(\frac{1-t}{2}\right) a+\left(\frac{1+t}{2}\right) b\right),\right. \\
\left.f\left(\left(\frac{1+t}{2}\right) a+\left(\frac{1-t}{2}\right) b\right), \frac{1}{2}\right) \leq 0,
\end{gathered}
$$

for all $t \in[0,1]$. Multiplying this inequality by $w(t)=\frac{\varphi\left(\left(\frac{b-a}{2}\right) t\right)}{t \Lambda(1)}$ and using axiom (A3), we get

$$
\begin{gathered}
F\left(\frac{\varphi\left(\left(\frac{b-a}{2}\right) t\right)}{t \Lambda(1)} f\left(\frac{a+b}{2}\right), \frac{\varphi\left(\left(\frac{b-a}{2}\right) t\right)}{t \Lambda(1)} f\left(\left(\frac{1-t}{2}\right) a+\left(\frac{1+t}{2}\right) b\right),\right. \\
\left.\frac{\varphi\left(\left(\frac{b-a}{2}\right) t\right)}{t \Lambda(1)} f\left(\left(\frac{1+t}{2}\right) a+\left(\frac{1-t}{2}\right) b\right), \frac{1}{2}\right)+L_{w(t)} \leq 0
\end{gathered}
$$


for all $t \in(0,1)$. Integrating over $(0,1)$ with respect to the variable $t$ and using axiom (A1), we obtain

$$
\begin{aligned}
& F\left(\frac{f\left(\frac{a+b}{2}\right)}{\Lambda(1)} \int_{0}^{1} \frac{\varphi\left(\frac{(b-a)}{2} t\right)}{t} \mathrm{~d} t,\right. \\
& \quad \frac{1}{\Lambda(1)} \int_{0}^{1} \frac{\varphi\left(\frac{(b-a)}{2} t\right)}{t} f\left(\left(\frac{1-t}{2}\right) a+\left(\frac{1+t}{2}\right) b\right) \mathrm{d} t, \\
& \left.\quad \frac{1}{\Lambda(1)} \int_{0}^{1} \frac{\varphi\left(\frac{(b-a)}{2} t\right)}{t} f\left(\left(\frac{1+t}{2}\right) a+\left(\frac{1-t}{2}\right) b\right) \mathrm{d} t, \frac{1}{2}\right) \\
& +\int_{0}^{1} L_{w(t)} \mathrm{d} t \leq 0 .
\end{aligned}
$$

Using the facts that

$$
\begin{aligned}
& \int_{0}^{1} \frac{\varphi\left(\frac{(b-a)}{2} t\right)}{t} f\left(\left(\frac{1-t}{2}\right) a+\left(\frac{1+t}{2}\right) b\right) \mathrm{d} t \\
& =\int_{\frac{a+b}{2}}^{b} \frac{\varphi\left(x-\frac{a+b}{2}\right)}{x-\frac{a+b}{2}} f(x) \mathrm{d} x \\
& ={ }_{b-} I_{\varphi} f\left(\frac{a+b}{2}\right),
\end{aligned}
$$

and

$$
\begin{aligned}
& \int_{0}^{1} \frac{\varphi\left(\frac{(b-a)}{2} t\right)}{t} f\left(\left(\frac{1+t}{2}\right) a+\left(\frac{1-t}{2}\right) b\right) \mathrm{d} t \\
& =\int_{a}^{\frac{a+b}{2}} \frac{\varphi\left(\frac{a+b}{2}-x\right)}{\frac{a+b}{2}-x} f(x) \mathrm{d} x \\
& ={ }_{a+} I_{\varphi} f\left(\frac{a+b}{2}\right),
\end{aligned}
$$

we obtain

$$
\begin{aligned}
& F\left(f\left(\frac{a+b}{2}\right), \frac{1}{\Lambda(1)}{ }_{b-} I_{\varphi} f\left(\frac{a+b}{2}\right), \frac{1}{\Lambda(1)}{ }_{a+} I_{\varphi} f\left(\frac{a+b}{2}\right), \frac{1}{2}\right) \\
& +\int_{0}^{1} L_{w(t)} \mathrm{d} t \leq 0,
\end{aligned}
$$

which gives (22).

On the other hand, since $f$ is $F$-convex, we have

$$
F\left(f\left(\left(\frac{1+t}{2}\right) a+\left(\frac{1-t}{2}\right) b\right), f(a), f(b), t\right) \leq 0, \quad \forall t \in[0,1],
$$


and

$$
F\left(f\left(\left(\frac{1-t}{2}\right) a+\left(\frac{1+t}{2}\right) b\right), f(a), f(b), t\right) \leq 0, \quad \forall t \in[0,1] .
$$

Using the linearity of $F$, we get

$$
\begin{gathered}
F\left(f\left(\left(\frac{1+t}{2}\right) a+\left(\frac{1-t}{2}\right) b\right)+f\left(\left(\frac{1-t}{2}\right) a+\left(\frac{1+t}{2}\right) b\right)\right. \\
f(a)+f(b), f(a)+f(b), t) \leq 0, \quad \forall t \in[0,1]
\end{gathered}
$$

Applying the axiom (A3) for $w(t)=\frac{\varphi\left(\frac{(b-a)}{2} t\right)}{t \Lambda(1)}$, we obtain

$$
\begin{aligned}
& F\left(\frac{\varphi\left(\frac{(b-a)}{2} t\right)}{t \Lambda(1)} \times\left[f\left(\left(\frac{1+t}{2}\right) a+\left(\frac{1-t}{2}\right) b\right)+\right.\right. \\
& \left.\quad f\left(\left(\frac{1-t}{2}\right) a+\left(\frac{1+t}{2}\right) b\right)\right], \frac{\varphi\left(\frac{(b-a)}{2} t\right)}{t \Lambda(1)}[f(a)+f(b)], \\
& \left.\quad \frac{\varphi\left(\frac{(b-a)}{2} t\right)}{t \Lambda(1)}[f(a)+f(b)], t\right)+L_{w(t)} \leq 0
\end{aligned}
$$

for all $t \in(0,1)$. Integrating over $(0,1)$ and using axiom (A2), we have

$$
\begin{aligned}
T_{F, w} & \left(\int_{0}^{1} \frac{\varphi\left(\frac{(b-a)}{2} t\right)}{t \Lambda(1)} \times\left[f\left(\left(\frac{1+t}{2}\right) a+\left(\frac{1-t}{2}\right) b\right)+\right.\right. \\
& \left.f\left(\left(\frac{1-t}{2}\right) a+\left(\frac{1+t}{2}\right) b\right)\right] \mathrm{d} t \\
& f(a)+f(b), f(a)+f(b))+\int_{0}^{1} L_{w(t)} \mathrm{d} t \leq 0
\end{aligned}
$$

that is

$$
\begin{gathered}
T_{F, w}\left(\frac{1}{\Lambda(1)}\left[{ }_{a+} I_{\varphi} f\left(\frac{a+b}{2}\right)+{ }_{b-} I_{\varphi} f\left(\frac{a+b}{2}\right)\right],\right. \\
f(a)+f(b), f(a)+f(b))+\int_{0}^{1} L_{w(t)} \mathrm{d} t \leq 0 .
\end{gathered}
$$

The proof of Theorem 7 is completed.

Remark 4. If we take $\varphi(t)=t$ in Theorem 7 , then the inequalities (22) and (23) reduce to the inequalities (20) and (21) 
Remark 5. If we take $\varphi(t)=\frac{t^{\alpha}}{\Gamma(\alpha)}$ in Theorem 7, then we have the following inequalities for Riemann-Liouville fractional integrals

$$
\begin{aligned}
& F\left(f\left(\frac{a+b}{2}\right), \frac{2^{\alpha} \Gamma(\alpha+1)}{(b-a)^{\alpha}} J_{b^{-}}^{\alpha} f\left(\frac{a+b}{2}\right),\right. \\
& \left.\quad \frac{2^{\alpha} \Gamma(\alpha+1)}{(b-a)^{\alpha}} J_{a^{+}}^{\alpha} f\left(\frac{a+b}{2}\right), \frac{1}{2}\right)+\int_{0}^{1} L_{w(t)} \mathrm{d} t \leq 0,
\end{aligned}
$$

and

$$
\begin{gathered}
T_{F, w}\left(\frac{2^{\alpha} \Gamma(\alpha+1)}{(b-a)^{\alpha}}\left[J_{b^{-}}^{\alpha} f\left(\frac{a+b}{2}\right)+J_{a^{+}}^{\alpha} f\left(\frac{a+b}{2}\right)\right],\right. \\
f(a)+f(b), f(a)+f(b))+\int_{0}^{1} L_{w(t)} \mathrm{d} t \leq 0,
\end{gathered}
$$

where $w(t)=\alpha t^{\alpha-1}$ which is given by Budak et al. in [5].

Corollary 2. If we take $\varphi(t)=\frac{t^{\frac{\alpha}{k}}}{k \Gamma_{k}(\alpha)}$ in Theorem 7, then we have the following inequalities for $k$-Riemann-Liouville fractional integrals:

$$
\begin{aligned}
F\left(f\left(\frac{a+b}{2}\right), \frac{2^{\frac{\alpha}{k}} \Gamma_{k}(\alpha+k)}{(b-a)^{\frac{\alpha}{k}}} I_{b-, k}^{\alpha} f\left(\frac{a+b}{2}\right),\right. \\
\\
\left.\quad \frac{2^{\frac{\alpha}{k}} \Gamma_{k}(\alpha+k)}{(b-a)^{\frac{\alpha}{k}}} I_{a+, k}^{\alpha} f\left(\frac{a+b}{2}\right), \frac{1}{2}\right)+\int_{0}^{1} L_{w(t)} \mathrm{d} t \leq 0,
\end{aligned}
$$

and

$$
\begin{gathered}
T_{F, w}\left(\frac{2^{\frac{\alpha}{k}} \Gamma_{k}(\alpha+k)}{(b-a)^{\frac{\alpha}{k}}}\left[I_{a+, k}^{\alpha} f\left(\frac{a+b}{2}\right)+I_{b-, k}^{\alpha} f\left(\frac{a+b}{2}\right)\right],\right. \\
f(a)+f(b), f(a)+f(b))+\int_{0}^{1} L_{w(t)} \mathrm{d} t \leq 0
\end{gathered}
$$

where $w(t)=\frac{\alpha}{k} t^{\frac{\alpha}{k}-1}$.

Remark 6. One can obtain several results for convexity, $\varepsilon$-convexity, $h$-convexity, etc by special choice of the function $F$ in Theorems 6 and 7 .

\section{Conclusion}

In the development of this work, using the definition of $F$-convex functions some new Hermite-Hadamard type inequalities via generalized fractional integrals have been deduced. We also give several results capturing RiemannLiouville fractional integrals and $k$-Riemann-Liouville fractional integrals as special cases. The authors hope that these results will serve as a motivation for future work in this fascinating area. 


\section{REFERENCES}

[1] H. Budak, M.Z. Sarikaya, and M.K. Yildiz, Hermite-Hadamard type inequalities for F-convex function involving fractional integrals, Filomat, 32 (16) (2018), 5509-5518.

[2] H. Budak and M.Z. Sarikaya, On Ostrowski type inequalities for Fconvex function, AIP Conference Proceedings, 1833 (020057) (2017). https://doi.org/10.1063/1.4981705

[3] H. Budak, T. Tunç, and M.Z. Sarikaya, On Hermite-Hadamard type inequalities for F-convex functions, Miskolc Mathematical Notes, 20 (1) (2019), 169-191.

[4] H. Budak, M.A. Ali and A. Kashuri, Hermite-Hadamard type inequalities for F-convex functions involving generalized fractional integrals, Studia Universitatis Babeş-Bolyai Mathematica, in press.

[5] H. Budak, Refinements of Hermite-Hadamard inequality for trigonometrically $\rho$ convex functions, Mathematica Moravica, 23 (2) (2019), 87-96.

[6] B. Defnetti, Sulla strati cazioni convesse, Annali di Matematica Pura ed Applicata, 30 (1949), 173-183.

[7] S.S. Dragomir and C.E.M. Pearce, Selected topics on Hermite-Hadamard inequalities and applications, RGMIA Monographs, Victoria University, 2000.

[8] S.S. Dragomir and R.P. Agarwal, Two inequalities for differentiable mappings and applications to special means of real numbers and to trapezoidal formula, Applied Mathematics Letters, 11 (5) (1998), 91-95.

[9] R. Gorenflo and F. Mainardi, Fractional calculus: integral and differential equations of fractional order, Springer Verlag, Wien, 223-276, 1997.

[10] H. Hudzik and L. Maligranda, Some remarks on s-convex functions, Aequationes mathematicae, 48 (1994), 100-111.

[11] D.H. Hyers and S.M. Ulam, Approximately convex functions, Proceedings of the American Mathematical Society, 3 (1952), 821-828.

[12] A.A. Kilbas, H.M. Srivastava, and J.J. Trujillo, Theory and Applications of Fractional Differential Equations, North-Holland Mathematics Studies, 204 (2006).

[13] U.S. Kirmaci, Inequalities for differentiable mappings and applications to special means of real numbers and to midpoint formula, Applied Mathematics and Computation, 147 (2004), 91-95.

[14] O.L. Mangasarian, Pseudo-convex functions, SIAM Journal on Control and Optimization (SICON), 3 (1965), 281-290.

[15] S. Miller and B. Ross, An introduction to the Fractional Calculus and Fractional Differential Equations, John Wiley \& Sons, USA, 1993.

[16] P.O. Mohammed and M.Z. Sarikaya, Hermite-Hadamard type inequalities for Fconvex function involving fractional integrals, Journal of Inequalities and Applications, 2018 (359) (2018).

[17] J.E. Pečarić, F. Proschan, and Y.L. Tong, Convex functions, partial orderings and statistical applications, Academic Press, Boston, 1992.

[18] C.E.M. Pearce and J. Pecaric, Inequalities for differentiable mappings with application to special means and quadrature formula, Applied Mathematics Letters, 13 (2000), 51-55.

[19] I. Podlubni, Fractional Differential Equations, Academic Press, San Diego, 1999.

[20] B. T. Polyak, Existence theorems and convergence of minimizing sequences in extremum problems with restrictions, Doklady Mathematics, 7 (1966), 72-75. 
[21] B. Samet, On an implicit convexity concept and some integral inequalities, Journal of Inequalities and Applications, 2016 (2016), Article ID: 308, 16 pages.

[22] M.Z. Sarikaya and F. Ertuğral, On the generalized Hermite-Hadamard inequalities, Annals of the University of Craiova - Mathematics and Computer Science Series, 47 (1) (2020), 193-213.

[23] M.Z. Sarikaya, A. Saglam, and H. Yildirim, New inequalities of Hermite-Hadamard type for functions whose second derivatives absolute values are convex and quasiconvex, Welcome to the Website of the International Journal of Open Problems in Computer Science and Mathematics (IJOPCM), 5 (3) (2012), 1-14.

[24] M.Z. Sarikaya and N. Aktan, On the generalization some integral inequalities and their applications, Mathematical and Computer Modelling, 54 (9-10) (2011), 2175-2182.

[25] M.Z. Sarikaya, A. Saglam, and H. Yildirim, On some Hadamard-type inequalities for $h$-convex functions, Journal of Mathematical Inequalities, 2 (3) (2008), 335-341.

[26] M.Z. Sarikaya, E. Set, H. Yaldiz, and N. Basak, Hermite-Hadamard's inequalities for fractional integrals and related fractional inequalities, Mathematical and Computer Modelling, 57 (2013), 2403-2407.

[27] M.Z. Sarikaya, T. Tunç, and H. Budak, Simpson's type inequality for F-convex function, Facta Universitatis, Series: Mathematics and Informatics, 32 (5) (2017), 747-753.

[28] S. Varosanec, On h-convexity, Journal of Mathematical Analysis and Applications, 326 (1) (2007), 303-311.

Hüseyín Budak

Department of Mathematics

Faculty of Science and Arts

Düzce UnIVERsity

DÜZCE

TURKEY

E-mail address: hsyn.budak@gmail.com

\section{Pinar Kösem}

Department of Mathematics

Faculty of Science and Arts

DÜzCe University

DÜZCE

TURKEY

E-mail address: pinarksm18@gmail.com 